\title{
Plant Leaf Identification Using Color and Multi-scale Fractal Dimension
}

\author{
André R. Backes ${ }^{1}$ and Odemir M. Bruno ${ }^{2}$ \\ 1 Instituto de Ciências Matemáticas e de Computação (ICMC) \\ Universidade de São Paulo (USP) \\ Avenida do Trabalhador São-carlense, 400 \\ 13560-970 São Carlos SP Brazil \\ 2 Instituto de Física de São Carlos (IFSC) \\ Universidade de São Paulo (USP) \\ Avenida do Trabalhador São-carlense, 400 \\ 13560-970 São Carlos SP Brazil \\ backes@icmc.usp.br, \\ bruno@ifsc.usp.br
}

\begin{abstract}
The most extracted measures from plants are traditionally performed manually. This and the great biodiversity of species makes the taxonomical classification of plants a very complex and time-consuming task. In order to contribute to the plant species characterization, a novel approach based on color texture is proposed. Each color channel in a leaf texture is modeled as a surface, so the complexity in each channel, as well as in the interaction among these three color channels, can be measured using the Multi-scale fractal dimension. Results show the potential of this approach, which overcomes traditional color texture analysis methods, such as Chromaticity Moments and Gabor EEE descriptors.
\end{abstract}

Keywords: plant identification, complexity, multi-scale fractal dimension, color texture analysis.

\section{Introduction}

Taxonomical classification of different plant species is a difficult task as it involves the analysis of many organs, such as flowers, seeds, leaves and woody parts [1, 2]. Various of these characteristics are seasonal (e.g., flowers and fruits) and so they may not be present for studies. Otherwise, a leaf sample is easily collected and it is a characteristic present for most of the plants, independent of the season or age of the plant. In fact, even fossils of plants can be studied through the use of leaves.

However, plant leaf identification still is a difficult task as the leaf can be influenced by numerous factors (such as, levels of maturity, sun exposure, soil influence, climate or even environment), thus resulting in a wide pattern variations of the samples collected.

Among the visual attributes that can be used to characterize an image, texture is one of the most important as it describes the surface of an object in terms

A. Elmoataz et al. (Eds.): ICISP 2010, LNCS 6134, pp. 463-470, 2010.

(C) Springer-Verlag Berlin Heidelberg 2010 
of the distribution of pixels over a region. This pixel distribution depends on the characteristics of the material, such as reflectance and homogeneity, which constitutes the surface of object. Given the importance of the texture, many approaches have been developed along the years: second-order statistics [3, [4], spectral analysis [5 11], wavelet packets [12, 13] and fractals [14, 15].

Leaves textures, different from artificial or human-made textures, don't represent a detectable quasi-periodic structure, but a random and persistent pattern that results in a cloud like texture appearance [14, 16]. This makes the fractal approach an interesting alternative to perform its analysis.

In fact, the presented paper is an extension of the approach from [15], where the Bouligand-Minkowski fractal dimension method is applied for gray-scale texture characterization. We intend in this paper to include the color information present in the texture pattern, as it is well-known that measurement of color and texture in combination, rather than isolated color or texture, would provide better discriminating power.

We start this paper by describing how texture and color analysis are performed in terms of complexity analysis (Section 2). A multi-scale approach is also used at this step. In Section 3, an experiment using a leaf texture database is described. We use statistical analysis to evaluate our approach. The results are showed and discussed in Section 4, where a comparative analysis is also performed using traditional color texture analysis methods, while Section 5 concludes this paper.

\section{Color Texture Analysis Based on Fractal Dimension}

The fractal dimension is an interesting tool to characterize objects in terms of space occupation and self-similarity. Many papers report the use of this property of fractals objects to characterize real and abstract objects in terms of complexity [17-23].

Here, the proposed approach presented is an extension from [15], where the Bouligand-Minkowski fractal dimension method is applied for gray-scale texture characterization. The gray-scale approach considers a texture as a set $S \in R^{3}$ of Cartesian coordinates, where each element $s \in S, s=(y, x, z)$, is defined by the pixel coordinates $(y$ and $x$ ) and the pixel intensity $z$, thus resulting in a surface in $R^{3}$ which represents the gray-scale texture. From this surface, it is possible to compute its influence volume $V(r)$ as

$$
V(r)=\left\{s^{\prime} \in R^{3}|\exists s \in S:| s-s^{\prime} \mid \leq r\right\},
$$

where $r$ is the dilatation radius, $s^{\prime}=\left(x^{\prime}, y^{\prime}, z^{\prime}\right)$ is a point in $R^{3}$ whose distance from $s=(x, y, z)$ is smaller or equal to $r$. Thus, the Bouligand-Minkowski fractal dimension $D$ is defined as

$$
D=3-\lim _{r \rightarrow 0} \frac{\log V(r)}{\log r},
$$

where $D$ is a number within $[0 ; 3]$. 
Note that, as the gray-scale information, any color channel present in a color texture could be mapped as a surface in the $R^{3}$. In fact, it is also possible to map all color channel as a different surface in the $R^{3}$. These approaches enables us to study the complexity in each color channel, as well as the complexity in the interaction among all color channels (Figure 1).

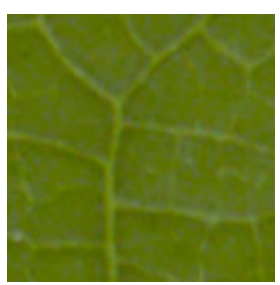

(a)

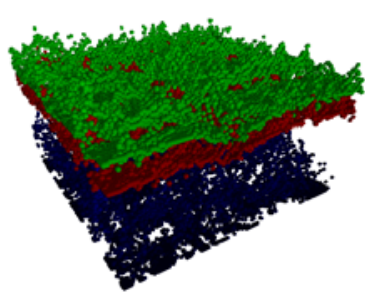

(b)

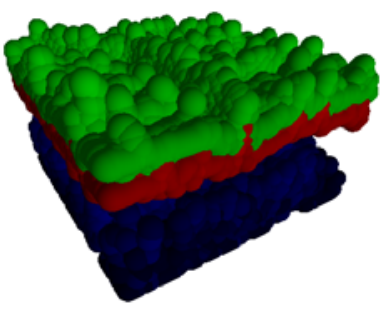

(c)

Fig. 1. (a) Original Texture; (b) Texture modelled as Cartesian coordinates; (c) Dilation using $r=5$

To provide a more accurate description of the color texture pattern, the MultiScale Fractal Dimension was used [18, 24, 25]. Instead of computing the line regression of the log-log curve $r \times V(r)$ to estimate the complexity, as performed in the fractal dimension method, this approach uses the derivative, thus exploiting the infinitesimal limit the linear interpolation. The result is a curve which expresses the complexity of an object in terms of the spatial scale (Figure 2):

$$
D(r)=3-\frac{d \log V(r)}{d \log r},
$$

where $D(r)$ represents the complexity of the object at scale $r$.

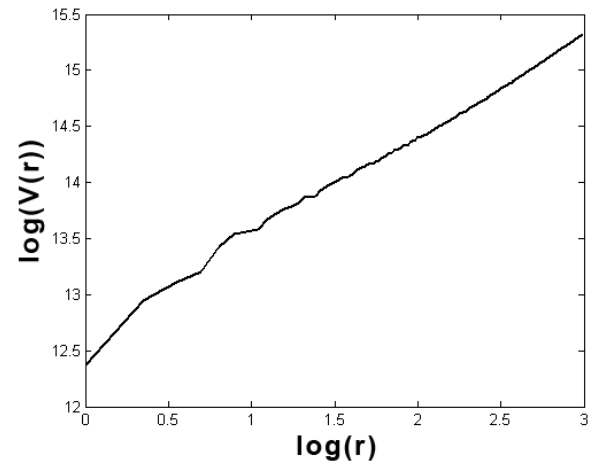

(a)

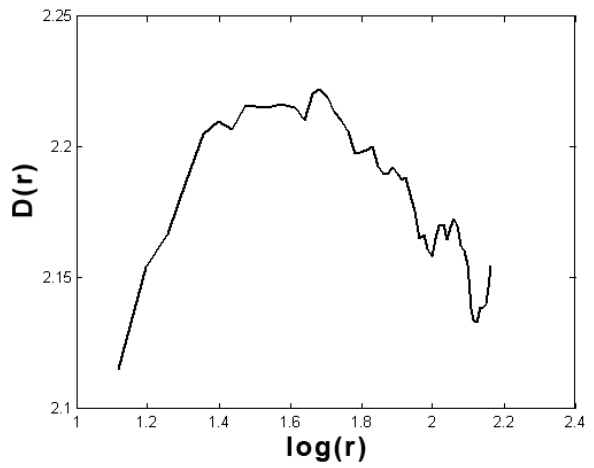

(b)

Fig. 2. (a) Log-log curve; (b) Multi-scale Fractal Dimension 


\section{Experiments}

Our proposed approach was evaluated by using a leaf database built using 10 leaves species from Brazilian flora. For each specie, 3 leaves samples were manually collected. The leaves samples were washed to prevent the presence of impurities, which could act as noise in the samples. A scanner using a 1200dpi (dots per inch) resolution was used. During the digitalization process, each leaf was oriented to have its central axis in a vertical position.

Five texture samples of $128 \times 128$ pixels of size were extracted from each leaf, thus resulting in a database composed by 150 texture samples grouped into 10 classes (Figure 3). Plant leaf identification is a difficult task due to the wide pattern variation of its fundamental features, such as color and texture (Figure 4). These variations are a response to different levels of maturity and sun exposure or other factors, such as soil influence, fungus, diseases, climate or even environment. Therefore, the extraction of the texture windows was performed manually in order to avoid texture patterns that do not characterize the real texture of that leaf specie.
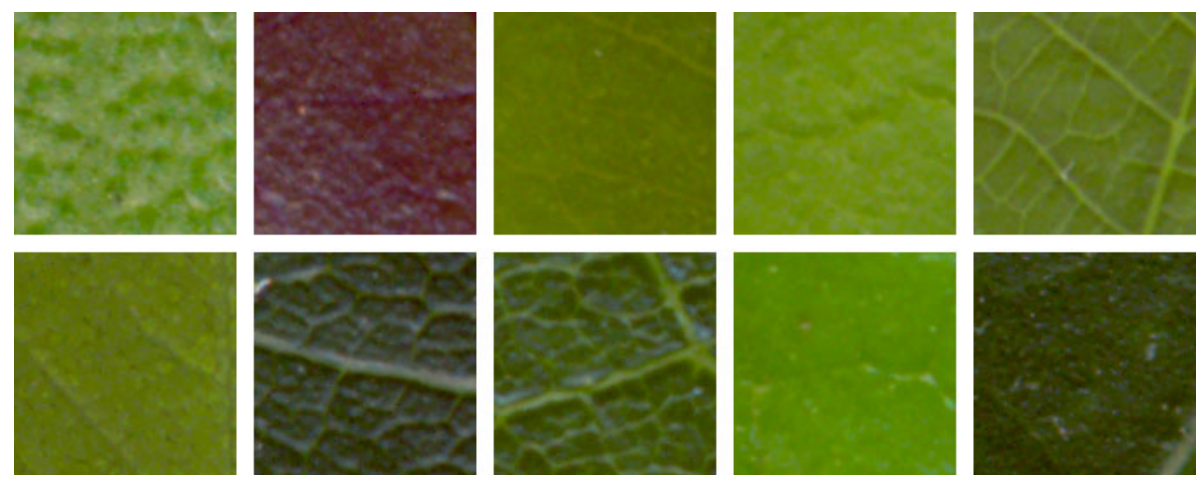

Fig. 3. Example of each texture class employed in the experiment

The supervised statistical classification method Linear Discriminant Analysis (LDA) 26, 27], in a leave-one-out cross-validation scheme, was used to evaluate the Multi-scale curve computed for each texture sample.

\section{Results}

During the experiments, the Multi-scale fractal dimension curves were computed considering a dilation radius $r=7$. The Finite Difference method [28] was used to compute the derivative. Two approaches were considered to characterize a color texture: (i) independent channels $(I C)$, where the descriptors are computed for each color channel and then combined to compose a feature vector for that color texture and (ii) dependent channels $(D C)$, where all channels are mapped into 

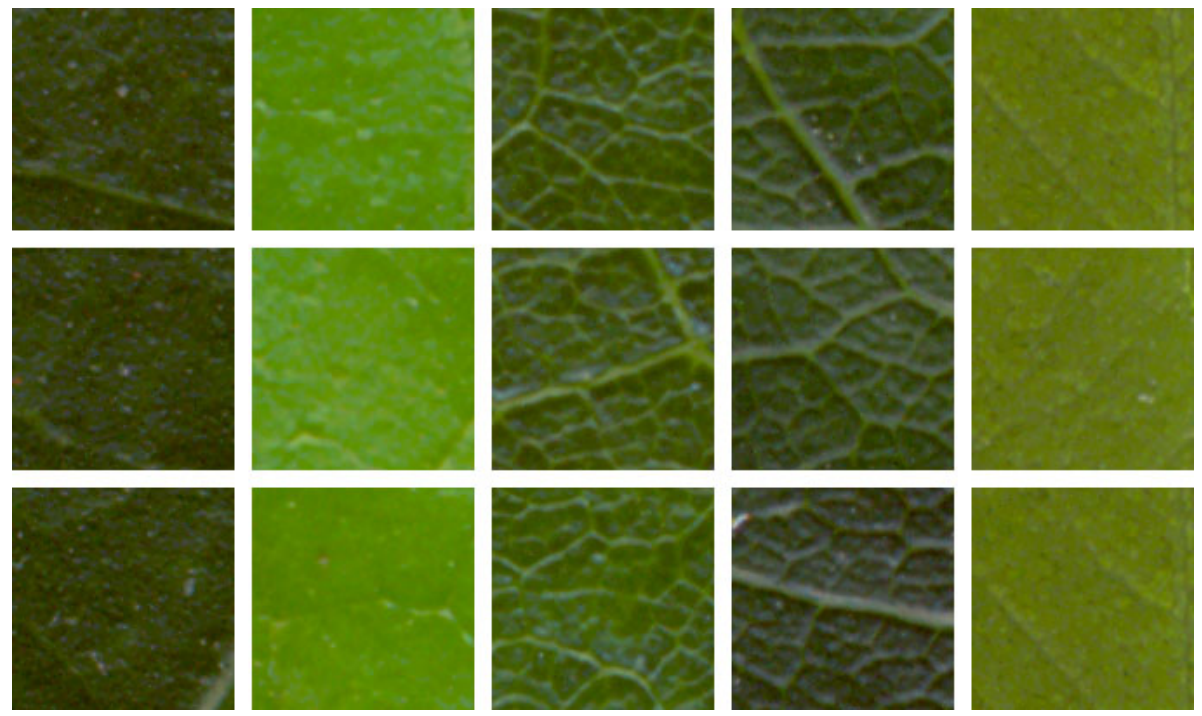

Fig. 4. Example of each texture class employed in the experiment. Example of texture variation in class (columns).

$R^{3}$ and dilated during the fractal dimension estimation process. This approach enables us to study the interaction among color channels, altogether.

A comparison between the proposed approach and traditional color texture analysis methods was also performed. Following, a brief description of the compared methods is presented:

Chromaticity Moments: this method is based on the concept of chromaticities as defined within the CIE XYZ color space. For each pixel in the image a pair of $(x, y)$ chromaticity values is computed. By analyzing the distribution of these chromaticity values, moments can be computed to compose a feature vector which enable us to characterize the image in terms of color and texture [29].

Gabor EEE: this method uses a linear transform from RGB to the Gaussian color model $\left(\tilde{E}, \tilde{E}_{\lambda}\right.$ and $\left.\tilde{E}_{\lambda \lambda}\right)$ over the color image [30, 31]. For each computed channel, a set of Gabor filters is applied. In the experiments, a total of 8 rotations and 8 scales, with a lower frequency of 0.01 and an upper frequency of 0.4 . The setup of the individual parameters of each filter follows the mathematical model presented in [9]. A total of 64 Gabor filters is built per channel, resulting in a feature vector with 192 features of the energy.

Table 1 shows the results yielded for each method considered. The first 50 descriptors were selected from Multi-scale curve to better describe a texture pattern in the $D C$ approach while only 14 were necessary in $I C$ approach. We also considerer the Multi-scale curves applied achieved from the gray scale version of the database. As we can see, the presence of color information improves the discriminant power of the signatures, thus providing a higher success rate. 
Table 1. Classification performance of different color texture descriptors

\begin{tabular}{|c|c|c|}
\hline Descriptor & Samples correctly classified & Success rate (\%) \\
\hline Gabor EEE & 144 & 96.00 \\
Chromaticity Moments & 104 & 69.33 \\
Multi-scale $I C$ & 146 & 97.33 \\
Multi-scale EEE $I C$ & 147 & 98.00 \\
Multi-scale DC & 144 & 96.00 \\
Multi-scale EEE DC & 135 & 90.00 \\
Multi-scale (gray) & 135 & 90.00 \\
\hline
\end{tabular}

We can note that the results of the $I C$ approach overcomes the ones from $D C$ approach. This is due to the fact that the evaluation of each color channel in an independent way provides more information about each color channel and, as a consequence, the whole texture pattern. On the other hand, the $D C$ approach enables us to perform color texture analysis in a single step. This is the great advantage, but it is also the main deficiency of this approach. Pixels with the same intensity value at different color channels are not as well represented in this approach as in the case where the color channels are separated.

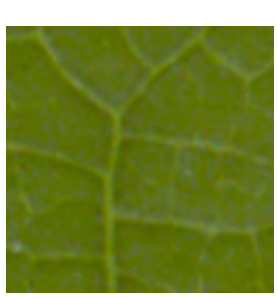

(a)

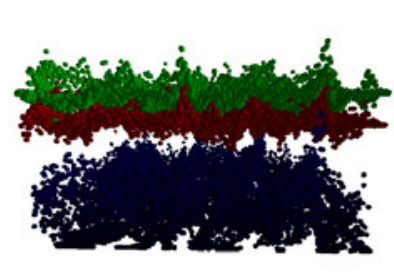

(b)

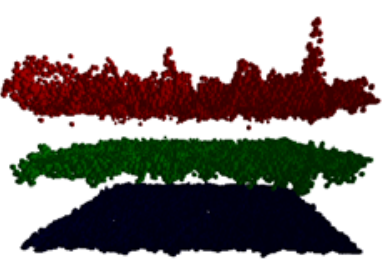

(c)

Fig. 5. (a) Original Texture; (b) Texture modelled as a surface; (c) Texture modelled as a surface after $E E E$ transformation

Results also show that the proposed approaches are more robust than the color texture methods compared, in special to the Gabor EEE method, which uses a color transformation to stress the characteristics of each channel. Thus, it is interesting to evaluate the proposed approaches using also this $E E E$ color transformation. As expected, the use of this transformation increases the success rate of the $I C$ approach. However, this transformation combines the information of the channels, thus leading to an equalization of the channels. This process diminishes the intersections among the color channels as it increases the distance among them (Figure 5). The information about the intersection is lost and the dilatation of each surface occurs almost independently in the $D C$ approach. As a result, the influence volume of the texture is basically the sum of the influence volume of each color channel, i.e., each color channel is computed as in the $I C$ approach, but their influence volumes are summed before the Multi-scale curve 
computing. In this case, the Multi-scale curve presents much less discriminant power than the use of descriptor computed for each channel, thus explaining its decrease in the success rate.

\section{Conclusion}

This paper presented a study of leaf classification based on the analysis of the complexity of its color texture pattern. We emphasize that the identification of leaf textures is a very difficult task, as it presents high similarity inter-classes and low similarity of intra-classes. The complexity analysis was performed using the Bouligand-Minkowski method and the Multi-scale Fractal Dimension. These methods present great sensitiveness to changes in the texture behavior, thus allowing the study of the pixels organization in the texture, as well as, its structural changes in terms of complexity along the scale. The achieved descriptors were evaluated in an experiment using linear discriminant analysis to classify a set of leaf texture previously selected. A comparison with traditional color texture analysis methods was also performed, and the results show the great potential of the proposed approach for natural texture analysis applications.

\section{Acknowledgments}

A.R.B. acknowledges support from FAPESP (2006/54367-9). O.M.B. acknowledges support from CNPq (306628/2007-4).

\section{References}

1. Judd, W., Campbell, C., Kellog, E.A., Stevens, P.: Plant Systematics: A Phylogenetic Approach. Sinauer Associates, Massachusetts (1999)

2. Kurmann, M.H., Hemsley, A.R.: The Evolution of Plant Architecture. Royal Botanic Gardens, Kew (1999)

3. Haralick, R.M.: Statistical and structural approaches to texture. Proc. IEEE 67(5), 786-804 (1979)

4. Murino, V., Ottonello, C., Pagnan, S.: Noisy texture classification: A higher-order statistics approach. Pattern Recognition 31(4), 383-393 (1998)

5. Shen, L., Bai, L.: A review on gabor wavelets for face recognition. Pattern Anal. Appl. 9(2-3), 273-292 (2006)

6. Bianconi, F., Fernández, A.: Evaluation of the effects of gabor filter parameters on texture classification. Pattern Recognition 40(12), 3325-3335 (2007)

7. Jain, A.K., Farrokhnia, F.: Unsupervised texture segmentation using Gabor filters. Pattern Recognition 24(12), 1167-1186 (1991)

8. Daugman, J., Downing, C.: Gabor wavelets for statistical pattern recognition. In: Arbib, M.A. (ed.) The Handbook of Brain Theory and Neural Networks, pp. 414-419. MIT Press, Cambridge (1995)

9. Manjunath, B.S., Ma, W.Y.: Texture features for browsing and retrieval of image data. IEEE Trans. Pattern Anal. Mach. Intell. 18(8), 837-842 (1996) 
10. Azencott, R., Wang, J.P., Younes, L.: Texture classification using windowed fourier filters. IEEE Trans. Pattern Anal. Mach. Intell. 19(2), 148-153 (1997)

11. Bajcsy, R.K.: Computer identification of visual surfaces. Computer Graphics Image Processing 2, 118-130 (1973)

12. Sengür, A., Türkoglu, I., Ince, M.C.: Wavelet packet neural networks for texture classification. Expert Syst. Appl. 32(2), 527-533 (2007)

13. Unser, M.: Texture classification and segmentation using wavelet frames. IEEE Trans. Image Processing 4(11), 1549-1560 (1995)

14. Kaplan, L.M.: Extended fractal analysis for texture classification and segmentation. IEEE Transactions on Image Processing 8(11), 1572-1585 (1999)

15. Backes, A.R., Bruno, O.M.: Plant leaf identification using multi-scale fractal dimension. In: Foggia, P., Sansone, C., Vento, M. (eds.) ICIAP 2009. LNCS, vol. 5716, pp. 143-150. Springer, Heidelberg (2009)

16. Huang, P.W., Dai, S.K., Lin, P.L.: Texture image retrieval and image segmentation using composite sub-band gradient vectors. J. Visual Communication and Image Representation 17(5), 947-957 (2006)

17. Chen, Y.Q., Bi, G.: On texture classification using fractal dimension. IJPRAI 13(6), 929-943 (1999)

18. de Plotze, R.O., Falvo, M., Pádua, J.G., Bernacci, L.C., Vieira, M.L.C., Oliveira, G.C.X., Bruno, O.M.: Leaf shape analysis using the multiscale minkowski fractal dimension, a new morphometric method: a study with passiflora (passifloraceae). Canadian Journal of Botany 83(3), 287-301 (2005)

19. Li, J., Sun, C., Du, Q.: A new box-counting method for estimation of image fractal dimension. In: International Conference on Image Processing, pp. 3029-3032 (2006)

20. da F. Costa, L., Jr., R.M.C.: Shape Analysis and Classification: Theory and Practice. CRC Press, Boca Raton (2000)

21. Carlin, M.: Measuring the complexity of non-fractal shapes by a fractal method. PRL: Pattern Recognition Letters 21(11), 1013-1017 (2000)

22. Schroeder, M.: Fractals, Chaos, Power Laws: Minutes From an Infinite Paradise. W. H. Freeman, New York (1996)

23. Tricot, C.: Curves and Fractal Dimension. Springer, Heidelberg (1995)

24. Emerson, C.W., Lam, N.N., Quattrochi, D.A.: Multi-scale fractal analysis of image texture and patterns. Photogrammetric Engineering and Remote Sensing 65(1), 51-62 (1999)

25. Gonzalez, R.C., Woods, R.E.: Digital Image Processing, 2nd edn. Prentic-Hall, New Jersey (2002)

26. Everitt, B.S., Dunn, G.: Applied Multivariate Analysis, 2nd edn. Arnold (2001)

27. Fukunaga, K.: Introduction to Statistical Pattern Recognition, 2nd edn. Academic Press, London (1990)

28. Smith, G.D.: Numerical Solution of Partial Differential Equations: Finite Difference Methods, 3rd edn., Oxford (1986)

29. Paschos, G.: Fast color texture recognition using chromaticity moments. Pattern Recognition Letters 21(9), 837-841 (2000)

30. Hoang, M.A., Geusebroek, J.M.: Measurement of color texture. In: Workshop on Texture Analysis in Machine Vision, pp. 73-76 (2002)

31. Hoang, M.A., Geusebroek, J.M., Smeulders, A.W.M.: Color texture measurement and segmentation. Signal Processing 85(2), 265-275 (2005) 